Ciência e Natura, v. 37 Part 2 2015, p. 406-414

\title{
ciênciaenatura
}

ISSN impressa: 0100-8307 ISSN on-line: 2179-460X

\section{Design of Intelligent Controllers for Double Inverted Pendulum}

\author{
Mehdi Ramezanifard
}

Electrical \& Electronic Engineering Department, Velayat University, Iranshahr, Iran

\begin{abstract}
In this paper, first a fuzzy controller is proposed for systems comprising two inverted pendulums placed in the same plane, where one pendulum moves along the X-axis and the other moves along the Y-axis; rules of a Fuzzy-Mamdani controller is improved by considering the behavior of an operator and a the developed expert system and by trial and error in the observation of the system's behavior. Simulation results obtained in MATLAB are proposed and then two other intelligent methods are presented. In the second proposed method, a TSK controller is designed with Anfis training approach and its simulation results are presented. Finally, a non-fuzzy controller (linearization feedback) is used as the supervisor fuzzy controller and the simulation results are presented. At last, performance of the three controllers is compared and the results are demonstrated.
\end{abstract}

Keywords: Double inverted pendulum, Fuzzy control, supervisory control, TSK controller, intelligent control. 


\section{Introduction}

S tability of a pendulum or an inverted pendulum is one of the basic problems of control engineering and several control solutions have already been proposed for it. The system comprises two inverted pendulums placed in the same plane; in fact it includes two separate inverted pendulum systems where one moves along the $\mathrm{X}$-axis and the other moves along the Y-axis [1]. Indeed, whether the cart can move along both axes is a separate discussion that has been resigned in this paper.

In this paper, control methods based on fuzzy logic have been used to stabilize the double inverted pendulum system. For this purpose, first the dynamic model of the inverted pendulum is obtained and then the controller is designed. It should be noted that in this system, there are two controllers with identical operation: one along the $\mathrm{X}$-axis and the other along the $\mathrm{Y}$-axis (indeed they may differ in terms of numerical values).

\section{Dynamic Model of the System together with Uncertainty}

Dynamic equations of the inverted pendulum system are as follows[2]:

$$
\begin{aligned}
& {\left[\begin{array}{l}
\dot{x}_{1} \\
\dot{x}_{2} \\
\dot{x}_{3} \\
\dot{x}_{4}
\end{array}\right]=\left[\begin{array}{c}
x_{2} \\
\frac{g \sin \left(x_{1}\right)-\frac{m}{2} l a x_{2}{ }^{2} \sin \left(2 x_{1}\right)}{\frac{4}{3} l-m l a \cos ^{2}\left(x_{1}\right)} \\
x_{4} \\
\frac{m}{2} a g \sin \left(2 x_{1}\right)+a l x_{2}^{2} \sin \left(x_{1}\right) \frac{4 m}{3} \\
\frac{4}{3}-m a \cos ^{2}\left(x_{1}\right)
\end{array}\right]+\left[\begin{array}{c}
0 \\
\frac{-a \cos \left(x_{1}\right)}{4} l-m l a \cos ^{2}\left(x_{1}\right) \\
0 \\
\frac{4 a}{3} \\
\frac{4}{3}-m a \cos ^{2}\left(x_{1}\right)
\end{array}\right]\left(u-\mu_{c} \operatorname{sign}\left(x_{4}\right)\right)} \\
& {\left[\begin{array}{llll}
x_{1} & x_{2} & x_{3} & x_{3}
\end{array}\right]^{T}=\left[\begin{array}{llll}
\theta & \dot{\theta} & x & \dot{x}
\end{array}\right]^{T}}
\end{aligned}
$$

Which as shown in Fig. 1:

1 is the length of the pendulum in meters, $\theta$ is the angle between the pendulum and the vertical axis in radians, $m$ is the mass in kilograms and $x$ is the movement along the axis ( $\mathrm{X}$ or $\mathrm{Y})$ in meters.

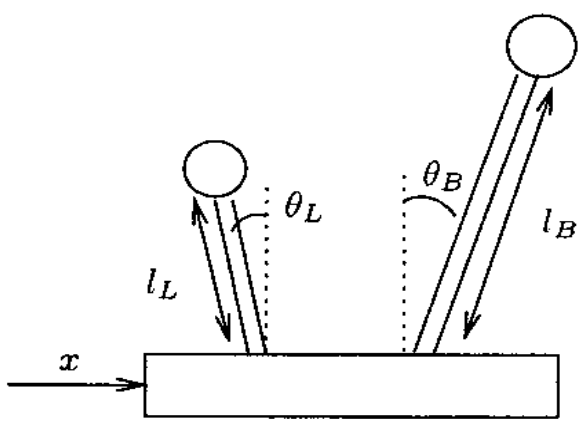

Fig.1. Inverted pendulum system model.

Uncertainty $\mu \mathrm{c}$ satisfies $0.004<\mu \mathrm{c}<0.006$. In this system, we have two separate model (like the above model), one for the inverted pendulum along the $\mathrm{X}$-axis and the other for the inverted pendulum along Y-axis.

\section{Design of a Fuzzy-Mamdani Controller}

First we define the membership functions of inputs and outputs as follows; inputs' domain is defined as $-0.6<\theta<0.6,-1<\dot{\theta}<1$, $-3<x<3,-6<\dot{x}<6$ and we define the fuzzy controllers as[8]:

pendulum $=$

name: 'pendulum'

type: 'mamdani'

andMethod: 'min'

orMethod: 'max'

defuzzMethod: 'centroid'

impMethod: 'min'

aggMethod: 'max'

input: [1x4 struct]

output: [1x1 struct]

rule: [1x81 struct]

Membership function's graph for both controllers is shown in Figures 2-4:

Fuzzy rules whose number changed to 81 after trial and error for enhancing the controller is defined as:

If (teta is $\mathrm{P}$ ) and (teta-dot is $\mathrm{P}$ ) and ( $\mathrm{x}$ is $\mathrm{N}$ ) and ( $x$-dot is $N$ ) then ( $u$ is VVN) (1)

If (teta is $\mathrm{P}$ ) and (teta-dot is $\mathrm{P}$ ) and ( $\mathrm{x}$ is $\mathrm{N}$ ) and $(x-\operatorname{dot}$ is $P)$ then $(u$ is $V N)(1)$ 


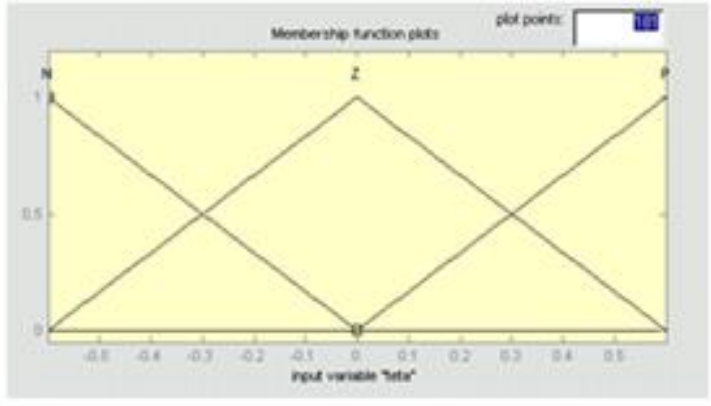

(A)

Fig.2. Membership functions of " $\theta$ "

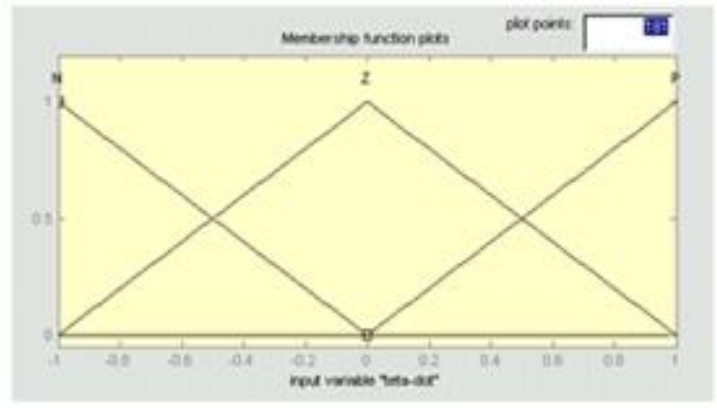

(B)

"(A) and " $\dot{\theta} "$ (B).

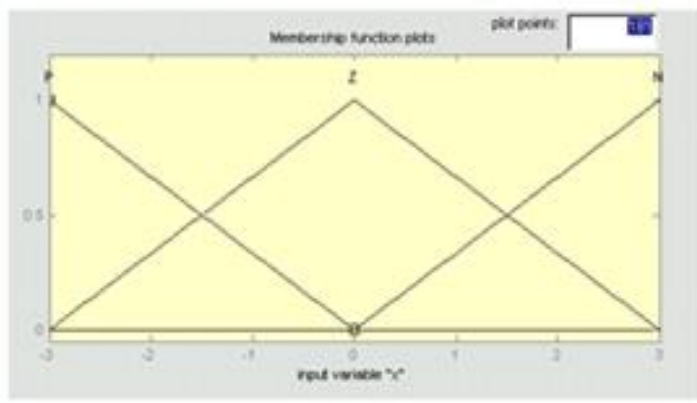

(A)

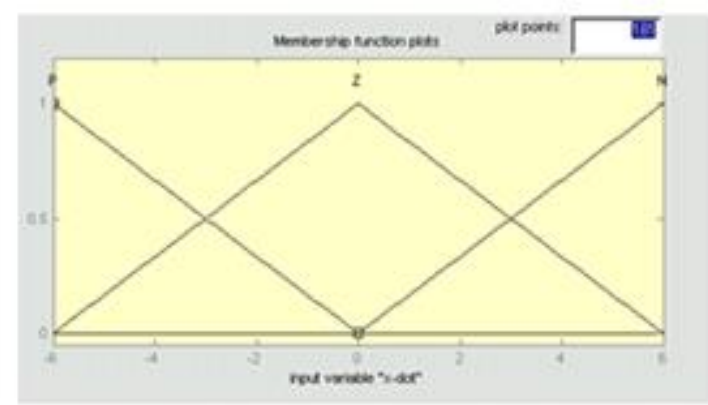

(B)

Fig.3. Membership functions of " $x$ " (A) and " $\dot{x}$ " (B).

If (teta is $\mathrm{P}$ ) and (teta-dot is $\mathrm{P})$ and ( $\mathrm{x}$ is $\mathrm{N}$ ) and ( $x$-dot is $Z$ ) then ( $u$ is VVN) (1)

If (teta is $P$ ) and (teta-dot is $P$ ) and ( $x$ is $P$ ) and $(x-\operatorname{dot}$ is $N)$ then ( $u$ is VVN) (1)

If (teta is $\mathrm{P}$ ) and (teta-dot is $\mathrm{P}$ ) and ( $\mathrm{x}$ is $\mathrm{P}$ ) and $(x-\operatorname{dot}$ is $P)$ then ( $u$ is VN) (1)

If (teta is $\mathrm{N}$ ) and (teta-dot is $\mathrm{Z}$ ) and ( $\mathrm{x}$ is $\mathrm{P}$ ) and ( $x$-dot is $P$ ) then ( $u$ is VP) (1)

If (teta is $N$ ) and (teta-dot is $Z$ ) and ( $x$ is $P$ ) and ( $x$-dot is $Z$ ) then ( $u$ is $P$ ) (1)

If (teta is $N$ ) and (teta-dot is $Z$ ) and ( $x$ is $Z$ ) and ( $\mathrm{x}-\operatorname{dot}$ is $N)$ then ( $\mathrm{u}$ is $\mathrm{Z})(1)$

If (teta is $N$ ) and (teta-dot is $Z$ ) and ( $x$ is $Z$ ) and ( $x$-dot is $P)$ then ( $u$ is VP) (1)

If (teta is $N$ ) and (teta-dot is $Z$ ) and ( $x$ is $Z$ ) and ( $x$-dot is $Z$ ) then ( $u$ is $P$ ) (1)

...

As seen, weight of all rules is equal to one.

\subsection{Simulation of Fuzzy-Mamdani Controller}

As mentioned before, we have two inverted pendulums along $\mathrm{X}$ and $\mathrm{Y}$ which are labeled number one and two respectively. Considering

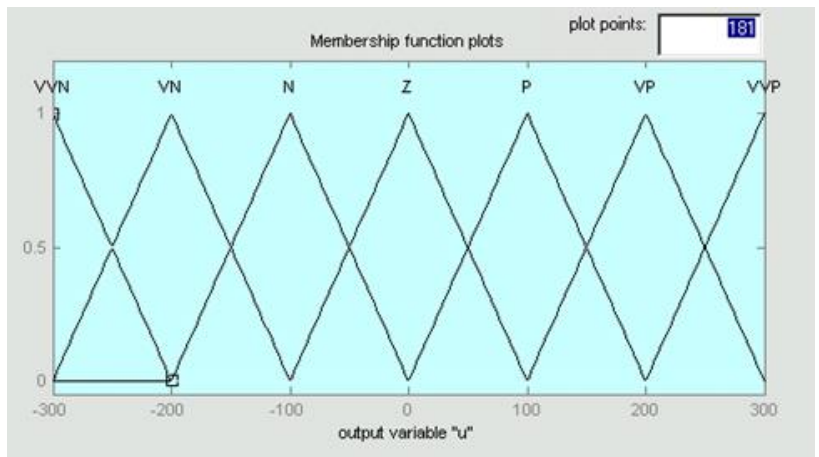

Fig.4. Membership functions of controller output " $u$ ".

$\mathrm{x} 1=0.55$ and $\mathrm{x} 2=-0.55$ radian and $\left(m_{1,2}=2, M=8, a=0.1, l_{1,2}=0.5, \mu_{c}=0.005, g=9.8\right)$, simulation results are shown in Figures 5-7. As seen, system has become stable:

\section{Design of a TSK Controller with Anfis Training Approach}

To generate data here, first data is generated by Fuzzy-Mamdani controller together with the system then the data in introduced to the Anfis network as input, after training, the controller is 


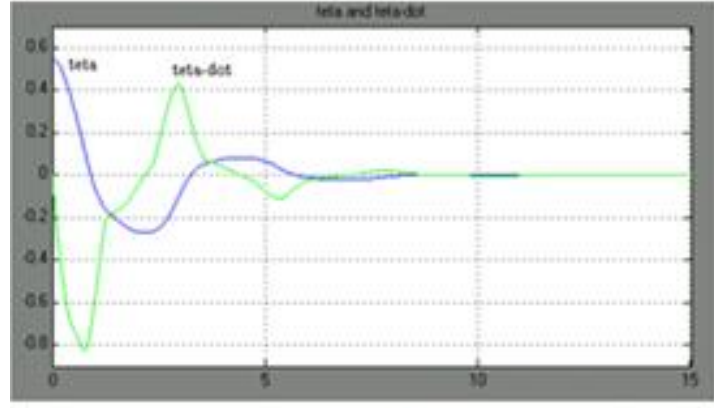

(A)

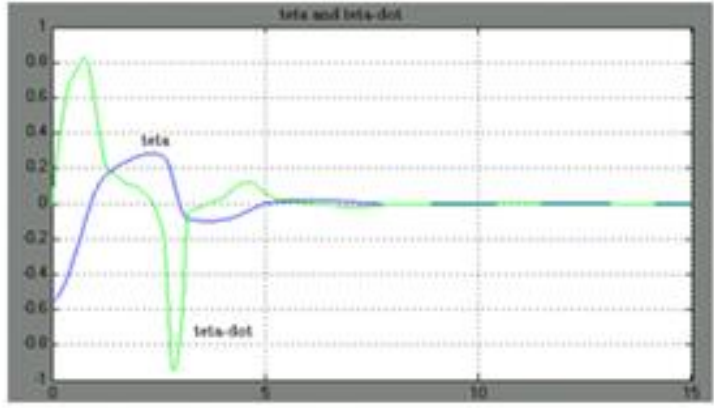

(B)

Fig.5. " $\theta$ " and " $\dot{\theta}$ " curves change, with Fuzzy controller, along the $\mathrm{X}$-axis (A) along the Y-axis (B).

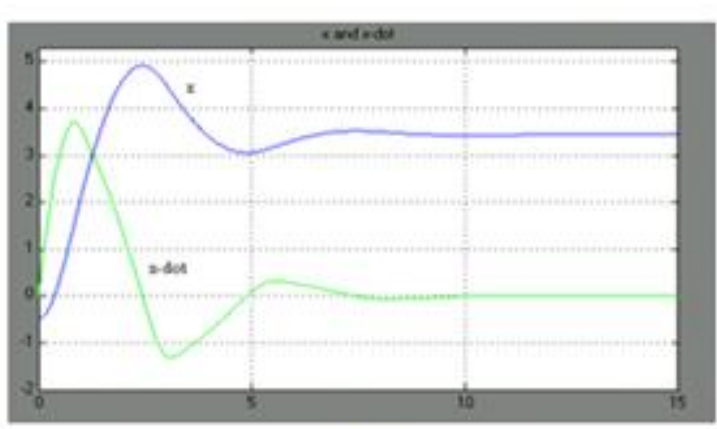

(A)

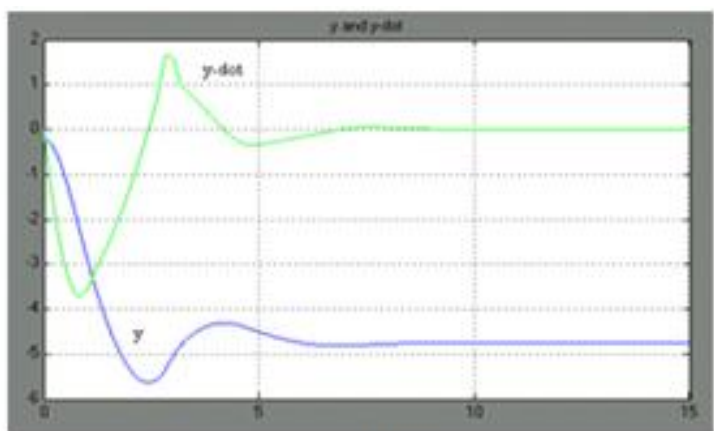

(B)

Fig.6. position and speed of cart curves changes, with Fuzzy controller, along the $\mathrm{X}$-axis (A) along the $\mathrm{Y}$-axis (B).

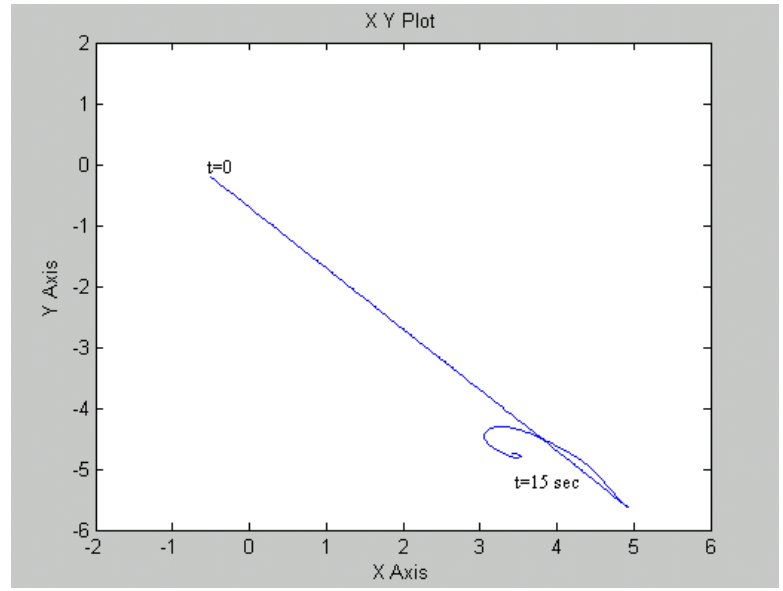

Fig.7. cart position in the $x-y$ plane (fuzzyMamdani method).

applied to the system and the results are obtained:

Penanfis $=$

$$
\begin{aligned}
& \text { name: 'penanfis' } \\
& \text { type: 'sugeno' } \\
& \text { andMethod: 'prod' } \\
& \text { orMethod: 'probor' } \\
& \text { impMethod: 'min' } \\
& \text { aggMethod: 'max' } \\
& \text { defuzzMethod: 'wtaver' } \\
& \text { input: [1x4 struct] } \\
& \text { output: [1x1 struct] } \\
& \text { rule: [1x1 struct] } \\
& \text { trndata: [751x5 double] }
\end{aligned}
$$

Membership function's graphs for each of the two Sugeno controllers are shown in Figures 811:

\subsection{Simulation}

Simulation results using TSK controller (with Anfis training approach), are shown in figures 12-14: 


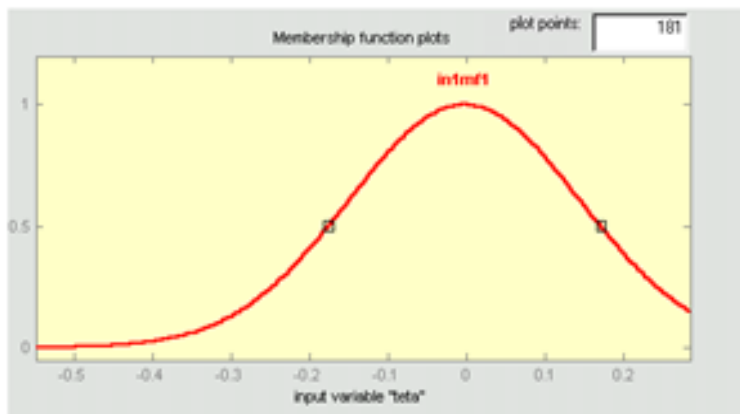

(A)

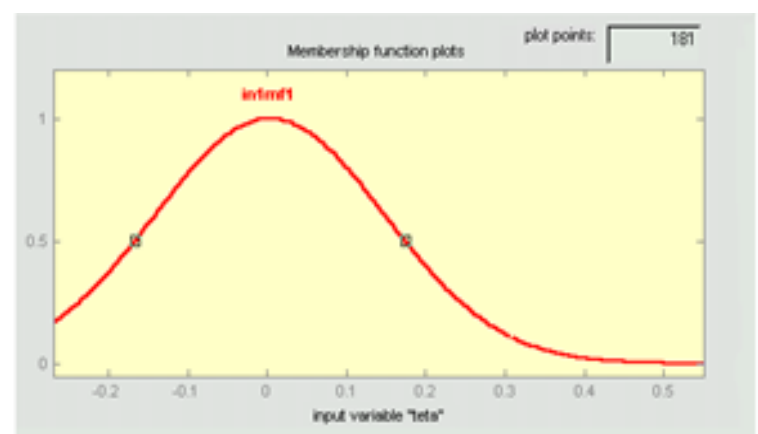

(B)

Fig.8. Membership functions of " $\theta$ ", $\mathrm{X}$-axis controller (A), Y-axis controller (B).

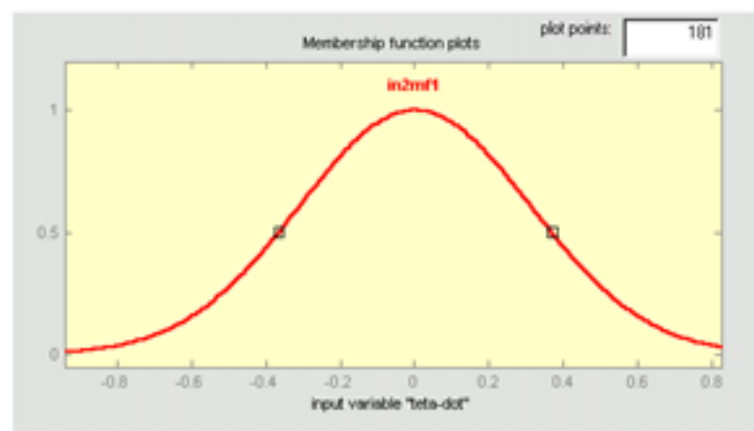

(A)

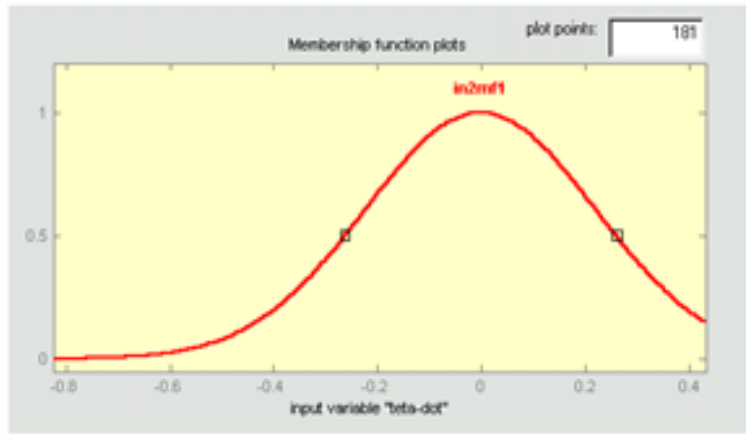

(B)

Fig.9. Membership functions of " $\dot{\theta}$ ", X-axis controller (A), Y-axis controller (B).

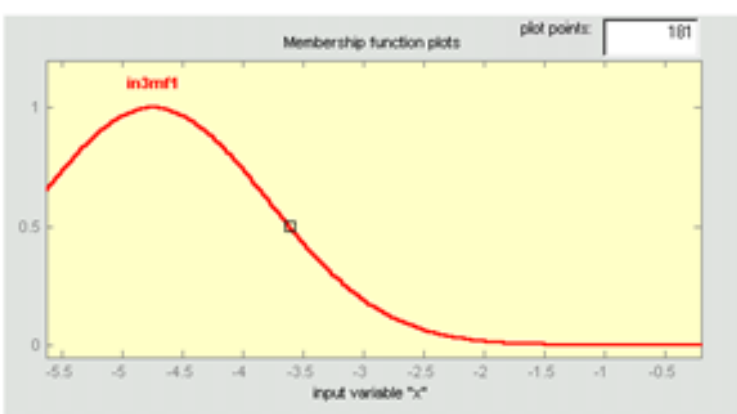

(A)

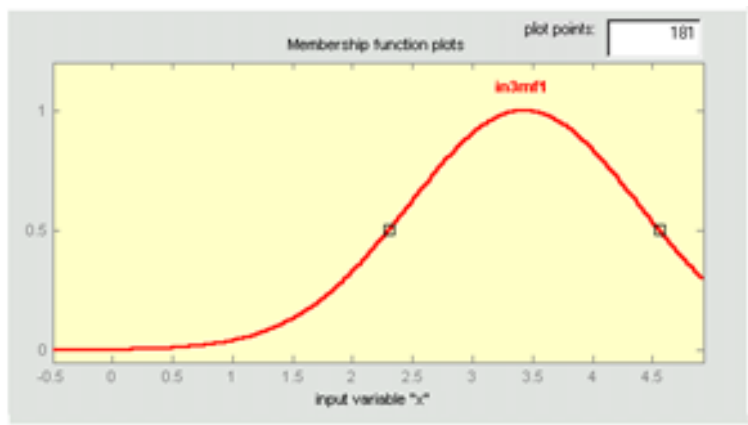

(B)

Fig.10. Membership functions of " $\mathrm{x}$ ", $\mathrm{X}$-axis controller (A), Y-axis controller (B).

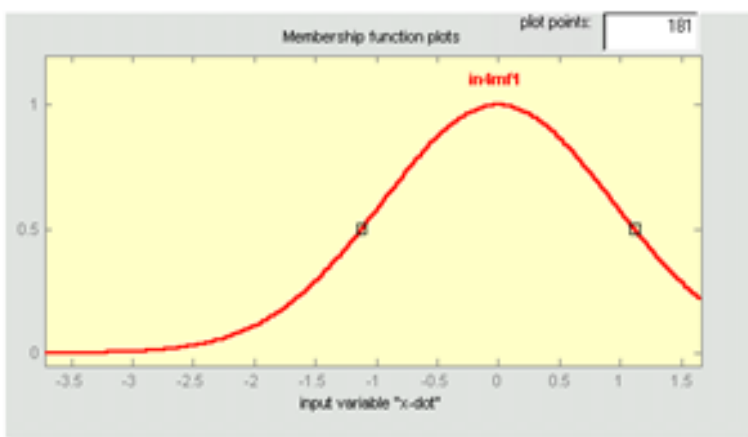

(A)

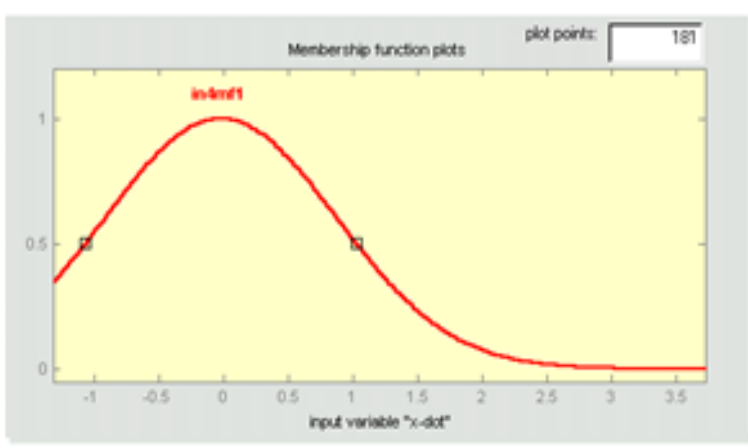

(B)

Fig.11. Membership functions of " $\dot{x}$ ", X-axis controller (A), Y-axis controller (B). 


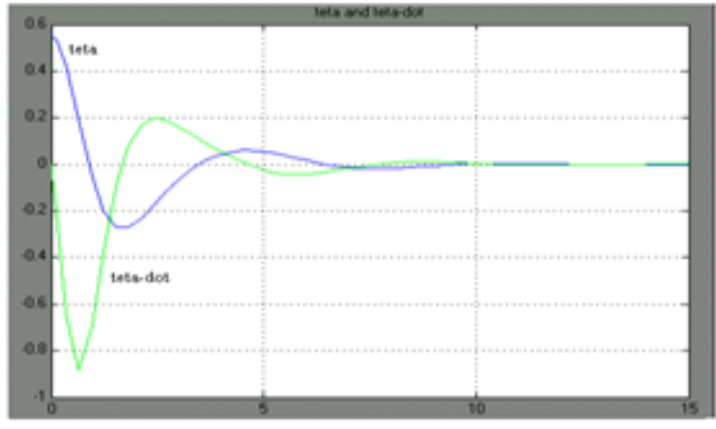

(A)

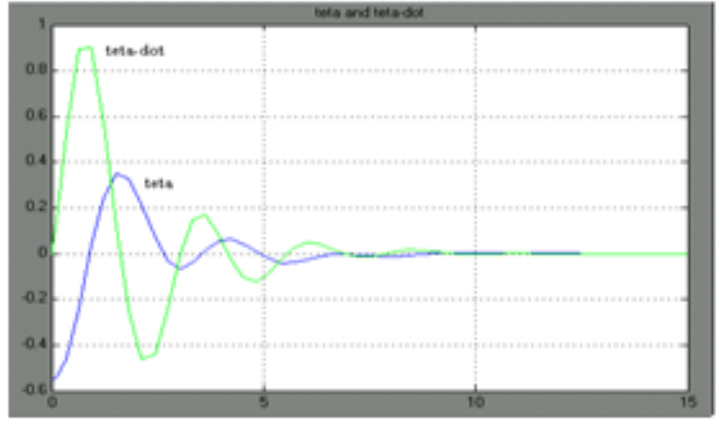

(B)

Fig.12. " $\theta$ " and " $\dot{\theta}$ " curves Change, with TSK controller (Anfis training approach), along the X-axis (A) along the $\mathrm{Y}$-axis (B).

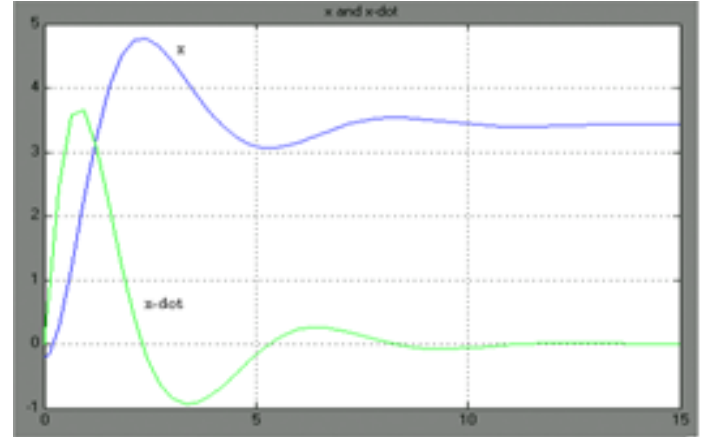

(A)

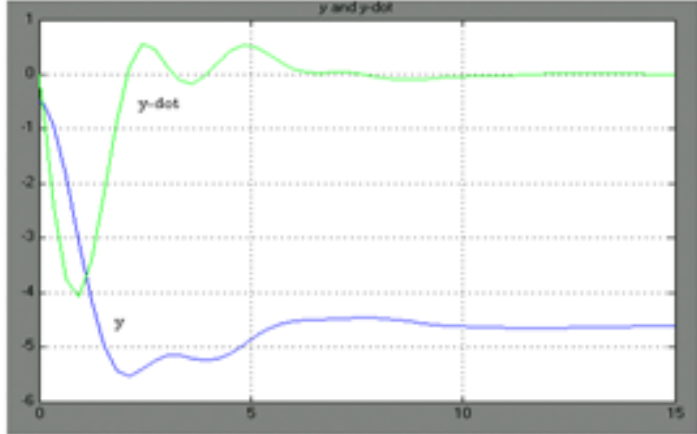

(B)

Fig.13. position and speed of cart curves Changes, with TSK controller (Anfis training approach), along the $\mathrm{X}$-axis (A) along the $\mathrm{Y}$-axis (B).

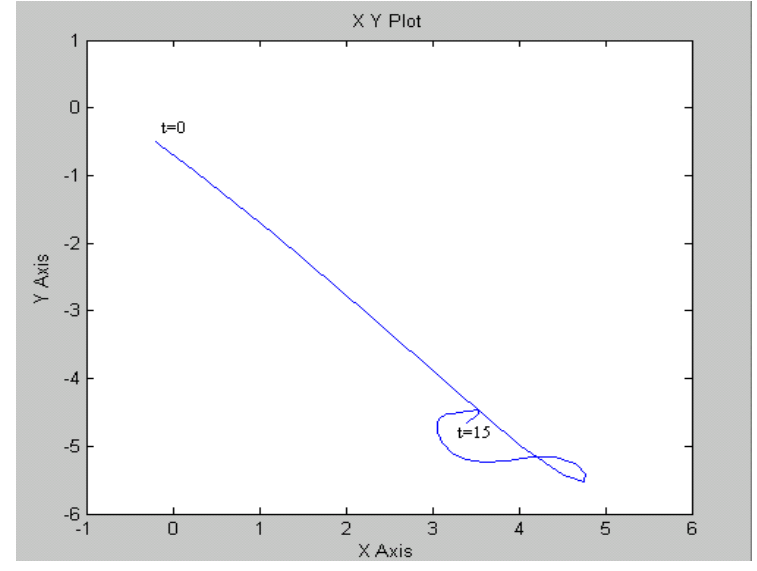

Fig.14. cart position in the $\mathrm{x}-\mathrm{y}$ plane (Anfis training approach).

As seen, TSK controller performs well with a relatively high speed but has steady state error same as Fuzzy-Mamdani controller.

\section{Design of a Fuzzy Controller with non-Fuzzy supervisor}

As implied in section 3; Fuzzy-Mamdani controller singly is able to stabilize and control the system for angles up-to maximum degrees, it is also seen that it does not perform well in terms of speed. In order to solve this problem, we have used an supervisory controller. A nonFuzzy supervisor (linearization feedback) is designed for the Fuzzy controller such that, if the inverted pendulum's angle exceeds a limit (23 degrees); supervisor controller takes action and reduces the angle immediately. In normal conditions (angles below 23 degrees) the fuzzy controller has to control the system. Thus not only the system becomes stable for a larger initial angle but also a simpler fuzzy controller (with less rules and inputs) is used, less calculation is required and the speed becomes higher.

It should be noted that in this section just the pendulum's angle is controlled and controlling the position and speed is resigned. Therefore, a Fuzzy-Mamdani controller with two inputs is used. Input's domain is defined as $-0.4<\theta<0.4, \quad-1<\dot{\theta}<1 . \quad$ Fuzzy-Mamdani controller is also defined as: 


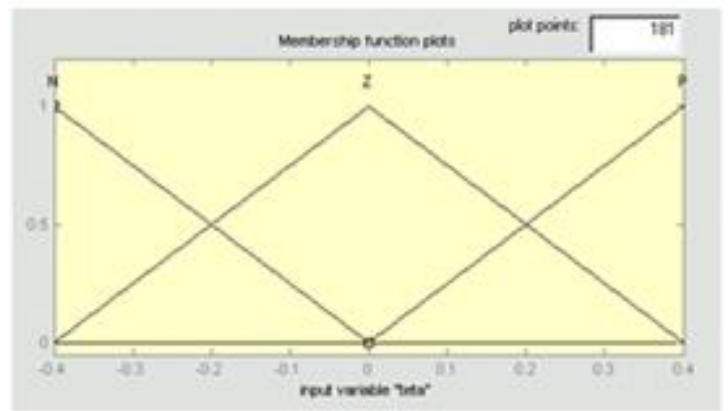

(A)

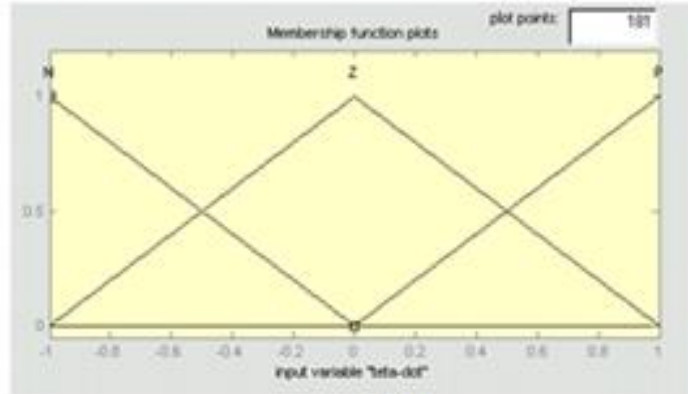

(B)

Fig.15. Membership functions of " $\theta$ " (A) and “ $\dot{\theta}$ " (B).

Pendulum2:

name: 'pendulum2'

type: 'mamdani'

andMethod: 'min'

orMethod: 'max'

defuzzMethod: 'centroid'

impMethod: 'min'

aggMethod: 'max'

input: [1x2 struct]

output: [1x1 struct]

rule: $[1 \times 9$ struct $]$

Input's and output's membership functions are defined as figures 15 and 16.

Nine fuzzy rules of this case are defined as follows:

If (teta is $\mathrm{P}$ ) and (teta-dot is $\mathrm{P}$ ) then ( $\mathrm{u}$ is $\mathrm{VN}$ ) (1)

If (teta is $P$ ) and (teta-dot is $N$ ) then ( $u$ is $Z$ ) (1)

If (teta is $P$ ) and (teta-dot is $Z$ ) then ( $u$ is $N$ ) (1)

If (teta is $N$ ) and (teta-dot is $P$ ) then ( $u$ is $Z$ ) (1)

If (teta is N) and (teta-dot is N) then ( $u$ is VP) (1)

If (teta is $N$ ) and (teta-dot is $Z$ ) then ( $u$ is $P$ ) (1)

If (teta is $Z$ ) and (teta-dot is $P$ ) then ( $u$ is $Z)(1)$

If (teta is $Z$ ) and (teta-dot is $N$ ) then ( $u$ is $Z$ ) (1)

If (teta is $Z$ ) and (teta-dot is $Z$ ) then ( $u$ is $Z$ ) (1)

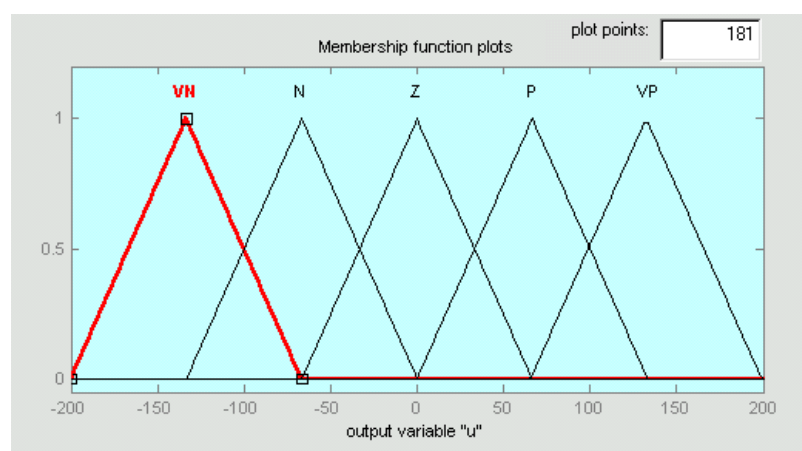

Fig.16. Membership functions of controller output "u".

\subsection{Design of the Supervisor Controller}

In supervisory control, at least, two levels as high-level (supervisor) and low-level controllers are used together to control a system under different conditions [7]. As stated before, a controller with linearization feedback is used as the supervisor in the supervisory control system. Equations of the supervisor controller are as follows.

Equations of the first controller (along $X$ ) and equations of the second controller (along $\mathrm{Y}$ ) are:

$\dot{x}_{1}=x_{2}$

$\dot{x}_{2}=f_{1}+b_{1} u$

$f_{1}=\frac{m_{t 1} g \sin \left(x_{1}\right)-m_{p 1} L x_{2}^{2} \sin \left(x_{1}\right) \cos \left(x_{1}\right)}{L\left(\frac{4}{3} m_{t 1}-m_{p 1} \cos ^{2}\left(x_{1}\right)\right)}$

$b_{1}=\frac{\cos \left(x_{1}\right)}{L\left(\frac{4}{3} m_{t 1}-m_{p 1} \cos ^{2}\left(x_{1}\right)\right)}$

$\dot{y}_{1}=y_{2}$

$\dot{y}_{2}=f_{1}^{\prime}+b_{1}^{\prime} u$

$f_{1}^{\prime}=\frac{m_{t 2} g \sin \left(y_{1}\right)-m_{p 2} L y_{2}^{2} \sin \left(y_{1}\right) \cos \left(y_{1}\right)}{L\left(\frac{4}{3} m_{t 2}-m_{p 2} \cos ^{2}\left(y_{1}\right)\right)}$

$b_{1}^{\prime}=\frac{\cos \left(y_{1}\right)}{L\left(\frac{4}{3} m_{t 2}-m_{p 2} \cos ^{2}\left(y_{1}\right)\right)}$

Where:

$\left(m_{p 1,2}=0.05, m_{c}=1, m_{t 1,2}=m_{p 1,2}+m_{c}, L=0.5, g=9.8\right)$ 
$u_{s}=\frac{V-f_{1}}{b_{1}}, V=-k_{1} \theta-k_{2} \dot{\theta}$

$\left(k_{1}=30, k_{2}=15, u=u_{f u z z}+I^{*} u_{s}\right)$

And

$$
I^{*}=\left\{\begin{array}{crr}
0 & ,|\theta|<0.38 \mathrm{rad} \\
\frac{|\theta|-0.38}{0.02} & , 0.38<|\theta|<0.4 & \mathrm{rad} \\
1 & ,|\theta|>0.4 & \mathrm{rad}
\end{array}\right.
$$

It should be mentioned that in the simulation, $\mathrm{mp}$ is the pendulum's mass, mc is the cart's mass and $\mathrm{mt}$ is the total mass. In order to determine the control input applied to the system in Fuzzy supervisory control with non-fuzzy supervisor approach, conditional variable $\mathrm{I}^{*}$ with above equations is used.

\subsection{Simulation of the Supervisory Controller}

Supervisor controller and fuzzy-mamdani controller are designed according to the above equations and the simulation results are shown in Figures 17 and 18.

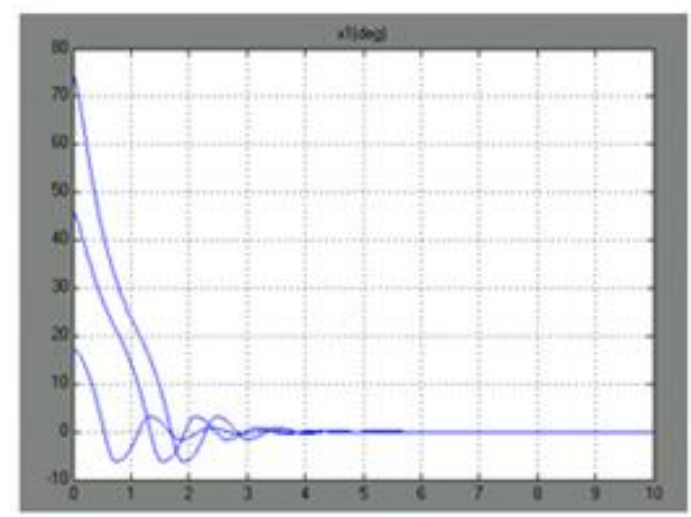

(A)
It can be seen that in this case, the controller is able to control and stabilize the system for its initial angle which is about \pm 70 degrees.

\section{Comparing the Controllers}

After reviewing the simulation results, controllers are compared in terms of accuracy, speed and robustness against uncertainty. Results of this comparison are given below:

- In terms of accuracy, all of the proposed controllers including fuzzy-mamdani controller, TSK controller and supervisory controller have steady state errors.

- In terms of speed, TSK controller has the highest speed, supervisory controller's speed is relatively good, but fuzzy-mamdani controller's speed is low.

- For studying the robustness against uncertainty, pendulum's mass is changed into various percentages of the initial mass and performance of the controllers is compared; it is seen that the three controller's performance is relatively the same and weak.

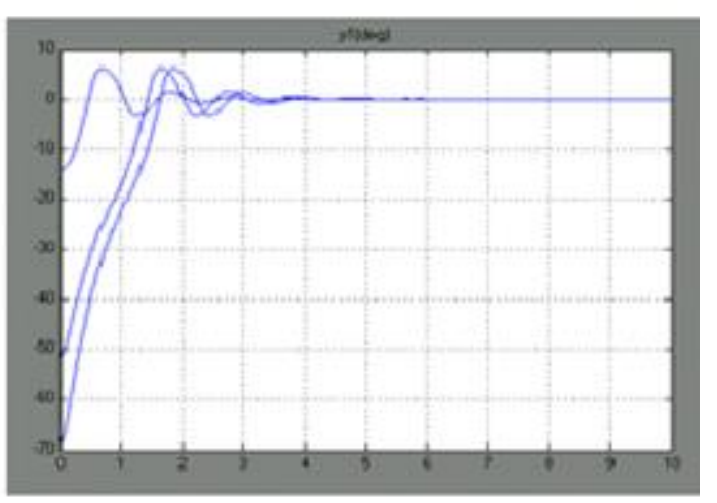

(B)

Fig.17. " $\theta$ ” curves change with different initial conditions in degree (supervisory controller), along the $\mathrm{X}$-axis (A) along the $\mathrm{Y}$-axis (B).

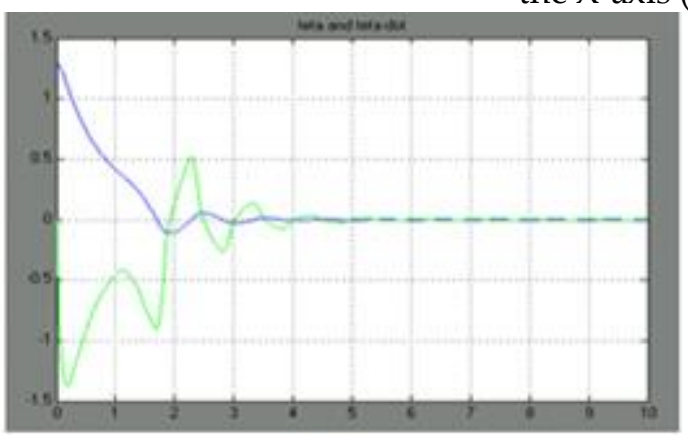

(A)

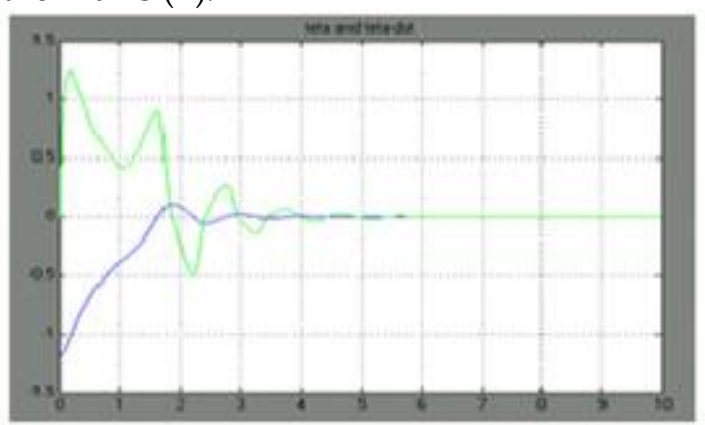

(B)

Fig.18. " $\theta$ " and " $\dot{\theta}$ " curves change, along the $\mathrm{X}$-axis (A) along the Y-axis (B). 


\section{Conclusion and Suggestions}

According to the comparison results in the previous section, all three controllers stabilize the system for initial conditions very well and the TSK controller (with Anfis training approach) has a higher speed. But all three controllers perform relatively poor against uncertainty.

Thus it is suggested to design other control approaches to enhance the robustness against uncertainty.

\section{References}

K.H. Lundberg and J.K. Roberge, "Classical Dual-Inverted-Pendulum Control", IEEE conference on Decision and Control, December,2003, pp.4399-4404.

Z. Matjaci'c and T. Bajd, "Arm-Free Paraplegic Standing-Part I: Control Model Synthesis and Simulation", IEEE TRANSACTIONS ON REHABI-LITATION ENGINEERING, VOL. 6, NO. 2, JUNE 1998, pp.125-138.

P. J. Larcombe, R. Z. The controllability of a double inverted pendulum by symbolic algebra analysis. Glasgow University, Proceedings of IEEE - Systems, Man and Cybernetics Conference (1993).

Jianqiang YI, Naoyoshi Yubazaki and K. Hirota. "Stabilization fuzzy control of parallel-type double inverted pendulum system", IEEE conference, 0-7803-5877-5, 2000.

M Ghane, M Zarvandi, MR Yousefi; Attenuating bullwhip effect using robust-intelligent controller, Intelligent Systems (IS), 2010 5th IEEE International Conference, 309-314

K. Ogata. Modern Control Engineering. Prentice Hall, 4 edition, 2001.

L.X. Wang, "A Course in Fuzzy Systems and Control".

M. Ramezanifard and A.R. Mohammad shahri, "Fuzzy Supervisory Control for mobile Robot Navigation in an Unknown Dynamic Environment", 14th International Conference of Electrical Engineering, Tehran, AmirKabir University of Technology, 2006
M. Ramezanifard, "Design and Comparison of Two intelligent Controllers for a Double Inverted Pendulum placed in the Same Plane". First National E-Conference of Technology Developments on Electrical, Electronics and Computer Engineering, 2015 